\title{
An X-Ray Diffractometer Cryostat Providing Temperature Control in the Range 4 to $300{ }^{\circ} \mathrm{K}^{*}$
}

\author{
Floyd A. Mauer and Leonard H. Bolz
}

(July 5, 1961)

\begin{abstract}
The diffractometer cryostat described earlier has been modified to provide a means for controlling temperature and for confining the vapor in equilibrium with volatile samples. Materials with vapor pressures as great as 1 atmosphere (atm) can be handled without loss of insulating vacuum, and temperatures can be controlled with an accuracy of $\pm 0.1^{\circ} \mathrm{K}$ in the range 4 to $20{ }^{\circ} \mathrm{K}$ decreasing to $\pm 1.0^{\circ} \mathrm{K}$ at $300^{\circ} \mathrm{K}$. Data on the lattice constant and thermal expansion of neon are given to demonstrate the suitability of the apparatusfor maintaining a desired temperature while working with a volatile material.
\end{abstract}

\section{Introduction}

In 1958 the authors collaborated with others in designing a cryostat for obtaining X-ray diffraction patterns of polycrystalline solids formed by deposition on a surface cooled by liquid helium [1]. ${ }^{1}$ It was used with some success in studying lowtemperature solids condensed from the gaseous phase, but the design imposed serious limitations on the choice of experimental conditions. The copper block on which samples were deposited was soldered directly to the bottom of the helium reservoir, and gaseous samples were introduced into the insulating vacuum space through a tube directed at this block. Most of the material froze where it first impinged on the helium-cooled surface, and could be examined by X-ray diffraction methods through thin beryllium windows in the outer wall. Deposition and examination at the boiling temperatures of conventional cryogenic liquids could be accomplished readily, but, at intermediate temperatures, observations could be made only while the block was warming after depletion of the refrigerant. The normal rate of warming $\left(1^{\circ}\right.$ per min) was too high to permit adequate $\mathrm{X}$-ray data to be obtained. It was not feasible to maintain a desired temperature by heating the block electrically while it was in contact with liquid helium, and when crude techniques for isolating and heating it were tried, the increase in vapor pressure of the sample usually interfered. When the vapor pressure exceeded $10^{-5} \mathrm{~mm}$ of $\mathrm{Hg}$, diffusion of the sample into the insulating vacuum spaces resulted in excessive heat transfer, while condensation on surfaces colder than the block eventually led to disappearance of the sample from the surface in the X-ray beam.

These difficulties have been overcome in recent modifications which permit the copper block to be isolated from the helium reservoir. It can be heated electrically or cooled at any time to the

*This work was performed under the National Bureau of Standards Free Radicals Research Program supported by the Department of Defense.

1 Figures in brackets indicate the literature references at the end of this paper. temperature of the refrigerant. An electronic controller maintains the desired temperature, and a Mylar window separates the specimen chamber from the insulating vacuum spaces so that sample vapor pressures as high as 1 atm can be tolerated. Moreover, heat leaks have been reduced so that 2 liters of liquid helium last for as long as $6 \mathrm{hr}$.

The method of isolating the copper block from the helium reservoir is an adaptation of that used by Henshaw [2] in his neutron diffractometer cryostat. In most other designs, a low-conductivity link is introduced between the sample and the liquid helium reservoir before an experiment is begun. Thereafter, the sample cannot be cooled to the temperature of the refrigerant and may require hours to reach its equilibrium temperature. Henshaw overcame these difficulties by placing his specimen block at the lower end of a thin-walled stainless steel tube joined to the bottom of the helium reservoir. Initially, liquid helium fills this tube and is in direct contact with the specimen block. When the block is to be heated, a plug is inserted in the top of the tube to exclude the liquid. Because of the low conductivity of the stainless steel used for the tube, the block can be heated to 40 or $50{ }^{\circ} \mathrm{K}$ before the increase in the evaporation rate of the liquid helium becomes excessive. Figure 1 shows how this design has been adapted to the diffractometer cryostat.

\section{Modification of the Cryostat}

By comparing figure 1 with the corresponding figure in the earlier paper [1] it can be seen that many features of the cryostat remain unchanged. It is a conventional double Dewar vessel consisting of a liquid helium vessel (21) surrounded by a liquid nitrogen container $(22)$ and radiation shields $(19,3)$ which screen the helium vessel from thermal radiation.

In modifying the cryostat the original copper block was detached from the helium reservoir at the soft solder joint (7). The inner vacuum wall (10), which had separated the vacuum around the helium 


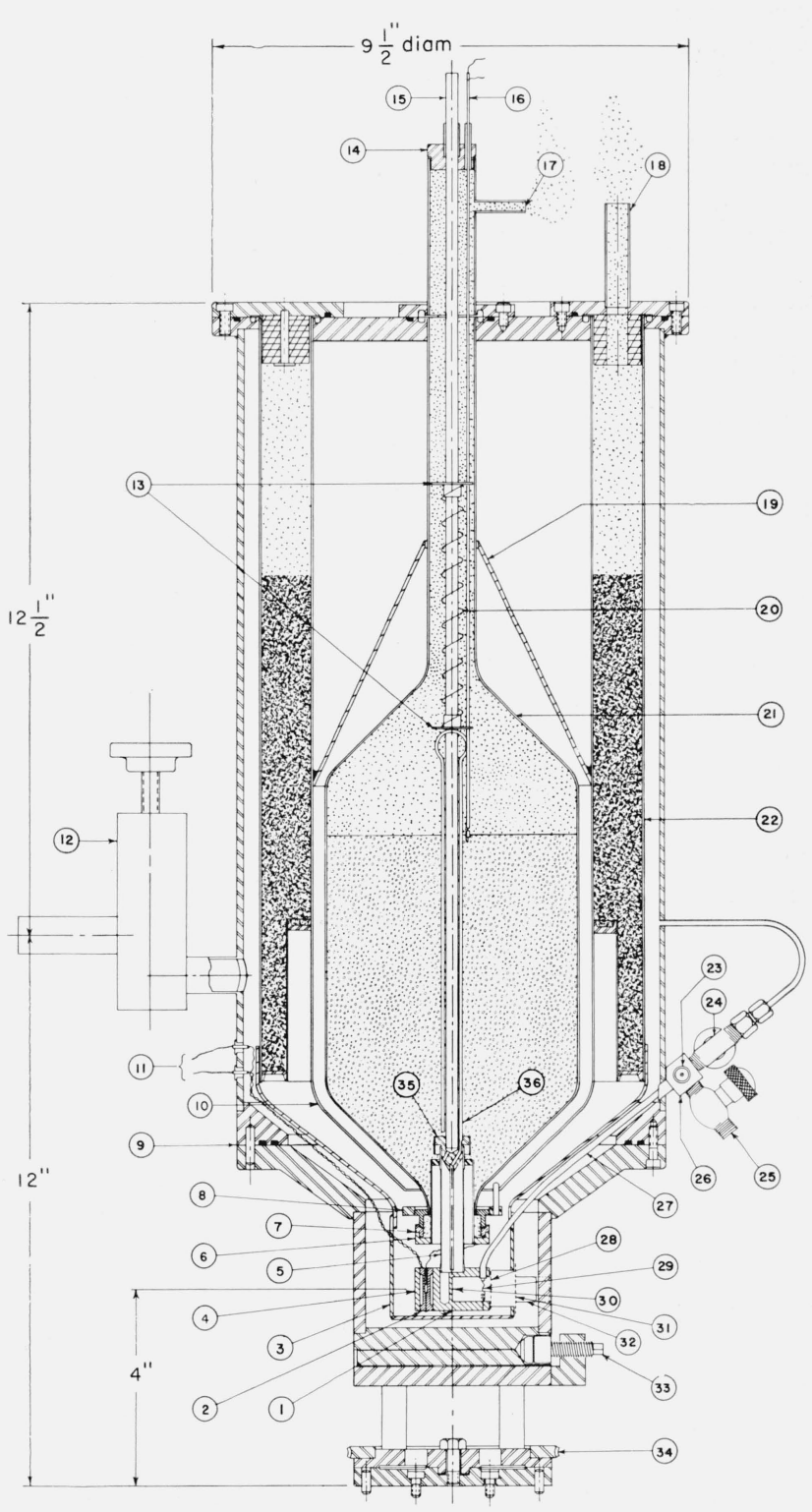

Figure 1. Vertical section through the X-ray diffractometer cryostat.

(1) Thermocouple, (2) heater bobbin, (3) radiation shield, (4) copper block, (5) thin-walled support tube (stainless steel), (6) copper ring, (7) soft-solder joint, (8) ring with centering pins, (9) flange with "o"-ring seal, (10) inner joint, (8) ring with centering pins, (9) lange with "o -ring seal, (10) baffle) (14) helium-vessel neck stopper, (15) block vent tube, (16) liquid-helium depth probe, (17) helium vent, (18) nitrogen fill-tube and vent, (19) radiation shield, probe, (17) helium vent, (23) inlet-tube(20) spacing spring, (21) helium vessel, (22) nitrogen vessel, 23 inlet-tubeheater terminal, (24) bypass valve, (25) sample-inlet valve, (26) manifold block, (27) sample-inlet tube (stainless steel), (28) Mylar plastic window, (29) sampleinlet-tube heater, (30) sample-deposition surface, ( 31 ) aluminum foil window
$(0.00035$-in. thick), (32) opening for 0.025-in, thick beryllium X-ray window, (33) translation-adjustment screw, (34) rotational-alinement gear sector, (35) plug, (36) internal siphon.

vessel from that in the specimen chamber, was then cut away from the helium vessel at the lower end to reduce heat conduction to the liquid helium. A ring (8) with three centering pins was fitted to the lower end of the inner vessel. The pins contact the remaining portion of the inner wall and prevent lateral motion. They do, however, allow for a vertical component of relative motion so that the two vessels may contract independently when filled with cold liquids.

Replacing the original copper block is an assembly which makes it possible to introduce the thin-walled tube (5) between the bottom of the helium reservoir and the copper block (4) without changing the location of the deposition surface (30) relative to the windows and X-ray beam. The copper ring (6) is fitted with a $7 / 8$-in. o.d. tube that extends for $13 / 4$-in. up inside the helium reservoir. At the top of this tube is a second ring which becomes, in effect, the new bottom of the liquid helium vessel. The $5 / 8$-in. o.d. tubing (5) of 304 stainless steel that supports the copper block is $2 \frac{1}{2}-\mathrm{in}$. long. The wall thickness, originally 0.035 in., has been reduced to 0.010 in. over a length of $2 \mathrm{in}$. to reduce the cross sectional area of the heat path. Because of the low thermal conductivity of 304 stainless steel $\left(\sim 2 \times 10^{-3}\right.$ watt $\left.\mathrm{cm}^{-1}{ }^{\circ} \mathrm{K}^{-1}\right)$, the heat flow along this path corresponding to a block temperature of $40^{\circ} \mathrm{K}$ is only about 0.025 watt.

The plug (35) which is inserted through the neck of the crvostat is used to exclude liquid helium from the region of the block when it is to be heated. A diagonal passage through this plug connects the internal siphon (36) with the tube extending to the bottom of the space below and permits liquid trapped in this region to return to the helium reservoir. Another passage connects this region with the vent tube (15). These passages may not be visible when the figure is reproduced.

A new sample deposition block (4) was cut from a cylinder (1 $1 / 2$-in. diam by $7 / 8$-in. high) of electrolytic tough pitch copper, selected for its high conductivity at low temperatures. A $1 / 2$-in. wide cut was made halfway through the cylinder, laaving a plane surface (30) on which samples are deposited. The block was gold-plated to provide a chemically inert surface that gives an X-ray pattern suitable for use as an internal standard.

The uncut portions of the cylinder above and below the deposition surface provide circular flanges around which the Mylar window (28) is cemented using an epoxy resin [3]. The window is $0.0007 \mathrm{in}$. thick and attenuates the copper $\mathrm{K}_{\alpha}$ radiation ordinarily used by only 8.5 percent. It is vacuumtight and readily supports a pressure differential of $1 \mathrm{~atm}$. The one failure of this window occurred at a pressure difference of about 3 atm.

A 23-ohm Manganin resistor wound on a copper bobbin (2) is provided for heating the block. The carbon resistor, (100 ohm, 1/10 watt) used as the sensing element for the temperature controller, is mounted in a $1 / 16-\mathrm{jn}$. hole drilled along the axis of the bobbin. The whole assembly is pressed into a $1 / 4$-in. hole in the copper block.

An inlet tube (27) connects the region enclosed by the Mylar window with the manifold (26) on the outside of the cryostat. Stainless steel tubing $1 / 8 \mathrm{in}$. in diameter with a 0.005 -in. wall thickness is used. 
To keep samples from freezing in this tube, it is lined with Teflon tubing through which is passed an elongated helix of \#30 Nichrome wire (29). The inner end is grounded to the copper block and the outer is connected to a terminal (23) in the manifold so that a current may be passed through the wire if blockage occurs.

The by pass valve (24) may be opened while pumping to equalize pressures on the two sides of the Mylar window, and closed before the inlet valve (25) is opened to admit the sample gas. Since the cryostat turns through $90^{\circ}$ while the X-ray pattern is scanned, flexible $1 / 8$-in. annealed copper tubing is used to connect this valve to the stationary specimen preparation train, flow gages, manometer, and auxilliary vacuum pump.

Other features that have not been described in the text are identified in the legend of figure 1.

\section{Temperature Controller}

Numerous controllers have been described in the literature [4], but the one used to control the temperature of the specimen block differs from all of these and will be described briefly. It uses the 100-ohm carbon resistor in the heater bobbin as the temperature sensing element (fig. 2). The resistor forms one arm of a Wheatstone bridge which is set to balance at a resistance corresponding to the desired temperature. The servo amplifier from a recording potentiometer amplifies the output of the bridge and drives a two-phase reversible motor to set a variable autotransformer. In this way, heater power input is automatically increased or decreased until the desired temperature is reached. To improve the stability of the system, a rate signal is obtained from a small $d$-c generator on the shaft of the balancing motor. While the system is approaching the control point, this generator produces a voltage proportional to the velocity. A portion of this voltage is fed back to the amplifier input, phased so that, when amplified, it tends to drive the motor in the reverse direction. Thus, a braking torque is provided that is very effective in reducing the amount of overshooting at the control point.

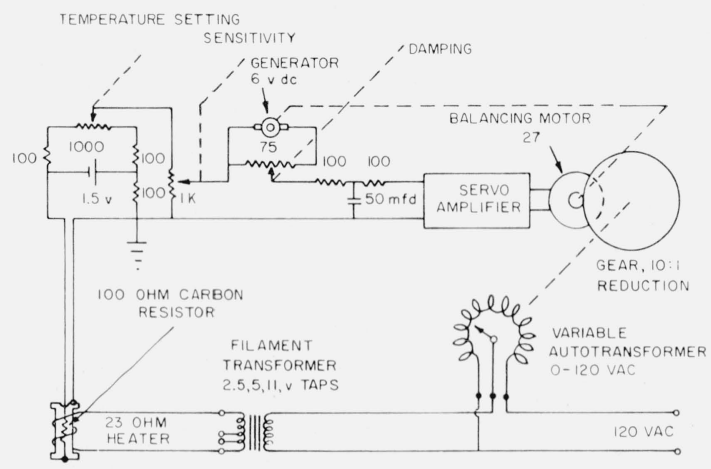

Figure 2. Temperature controller circuit.
In order to reduce the time required for the resistor to sense a change of power input, the compact $1 / 10$-watt size is used. It is cemented into a 1/16-in. hole in the heater bobbin. One lead is soldered directly to the block, and the other is cut very short to decrease its heat capacity.

A separate resistor (56 ohm, 1 watt) is mounted in the block and used for measuring temperatures in the range below $30^{\circ} \mathrm{K}$. In addition, a thermocouple $(\mathrm{Au}+2.11$ atomic \% Co versus $\mathrm{Cu})$ is used throughout the range 4.2 to $300{ }^{\circ} \mathrm{K}$.

The use of carbon resistors in low temperature thermometry has been described by Clement and Quinell [5]. Two characteristics of these thermometers are their extreme sensitivity at low temperatures and the rapid decrease of sensitivity with increasing temperature. Thus, by measuring resistance to 1 part in 5,000 , it is possible to detect temperature changes of $0.0001^{\circ}$ at $2{ }^{\circ} \mathrm{K}, 0.01{ }^{\circ} \mathrm{K}$ at $20^{\circ} \mathrm{K}$, or $1.0^{\circ} \mathrm{K}$ at $300^{\circ} \mathrm{K}$. In the present application, the inherent sensitivity cannot be fully utilized, and below $20^{\circ} \mathrm{K}$ the precision of control is limited to $\pm 0.1^{\circ} \mathrm{K}$. Above $20^{\circ} \mathrm{K}$ the precision gradually decreases to about $\pm 1^{\circ} \mathrm{K}$ at room temperature.

\section{Operation}

In order to fill the cryostat with liquid helium, the neck-stopper (14, fig. 1.) and the entire plug assembly are removed so that the transfer siphon can be inserted. When the inner vessel has been filled to approximately the level shown, the siphon is removed and the neck-stopper is replaced after drawing the plug (35) all the way up so that it remains in the neck tube to be cooled by the escaping vapor. The sample is usually introduced at this time by opening the inlet valve (25) and allowing the gas to flow in at a rate of 20 to $30 \mathrm{~cm}^{3} / \mathrm{min}$ (STP) until the thickness of the deposit is sufficient to reduce the intensity of the X-ray pattern from the gold substrate by a predetermined amount. When the block is to be heated, the plug is lowered and seated in the top of the tube (5) to exclude liquid helium. That trapped below the plug is returned to the reservoir through the internal siphon (36) by closing the vent (15) so that pressure develops in the well.

With helium out of the well, the block may be heated electrically, using the controller to maintain a desired temperature. The setting of the bridge is determined by referring to curves based on the semiempirical equation of Clement and Quinell for the calibration of carbon resistance thermometers. Experience soon indicates the best settings for the sensitivity and damping controls.

The relationship between power input and block temperature for three refrigerants is shown in figure 3 . Some heat reaches the block by conduction along the electrical leads and sample inlet tube even when the electrical power input is reduced to zero. As a result, the temperature of the block rises 10 to $20^{\circ}$ above the temperature of the refrigerant. The internal siphon (36, fig. 1.), which was not a part of the system described by Henshaw, was introduced 


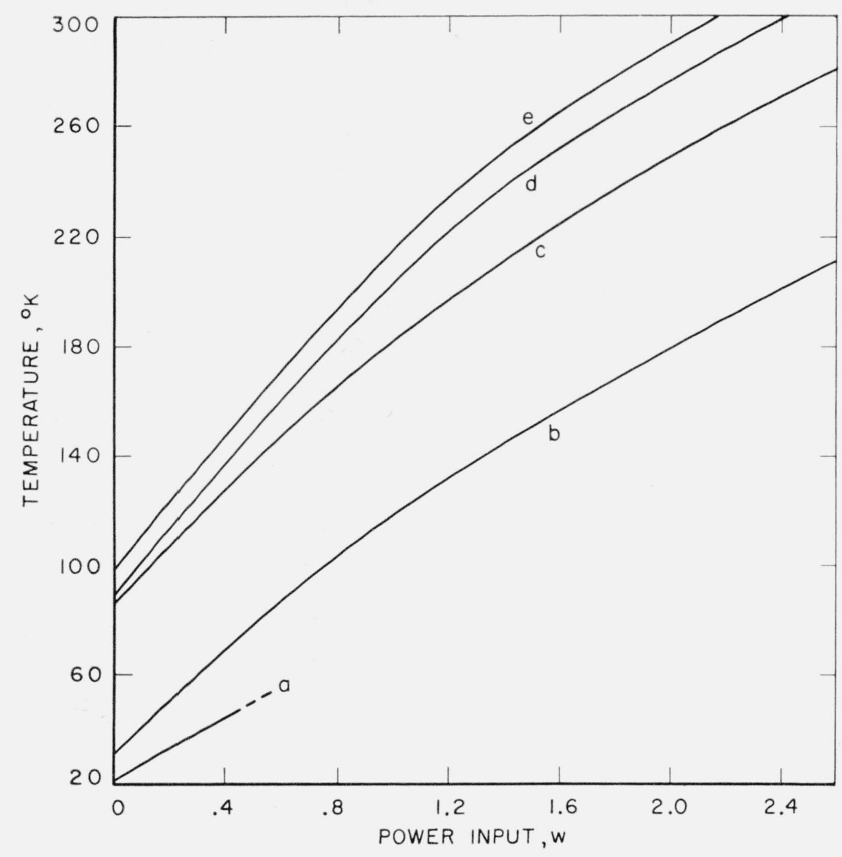

FIGURE 3. Variation of block temperature with heater power input for (a) liquid helium, (b) liquid hydrogen, and (c) liquid nitrogen.

For the last refrigerant, the effect of residual gas in the region below the plug is seen by comparing curve (c), obtained with nitrogen gas at $1 \mathrm{~atm}$ in the well, with curve (d), obtained by filling the region with styrofoam, and curve (e), obtained by evacuating the region to eliminate both conduction and convection.

in an attempt to control temperatures in the interval between the boiling point of the refrigerant and the zero-power intercept. By pumping on the block vent (15) through a needle valve, helium (for example) can be drawn in at a controlled rate. If desired, the automatic temperature controller may be used simultaneously. This method is effective down to 10 to $12^{\circ} \mathrm{K}$. At lower temperatures twophase flow evidently commences and results in discontinuous cooling.

The arrangement that has given the best results in the range 4.2 to $15^{\circ} \mathrm{K}$ is a sliding-piston plug which is substituted for the plug shown (35, fig. 1.). It was hoped that any desired temperature could be reached by sliding the plug up or down to vary the effective length of the heat path along the thinwalled tube (5). However, with the block vent (15) closed to create a positive pressure, keeping helium from leaking around the piston, the lowest temperature reached with the plug all the way down is 12 ${ }^{\circ} \mathrm{K}$. To reach lower temperatures it is necessary to provide an escape to the atmosphere for helium gas. The rate of leakage around the plug is determined by the rate at which gas is allowed to escape and this is easily controlled by a needle valve (not shown). Each full turn changes the block temperature by very roughly $0.5{ }^{\circ} \mathrm{K}$. The temperature remains constant $\left( \pm 0.1^{\circ} \mathrm{K}\right)$ for periods of $10 \mathrm{~min}$ or more, and automatic control is available if desired. The valve is simply opened enough to give the lowest temperature required and, thereafter, the controller is used to adjust the heater input.

\section{Application}

Temperature was controlled in this way to obtain data on the lattice constant of neon at various temperatures in the range 4.2 to $22{ }^{\circ} \mathrm{K}$ (fig. 4) [6]. Values of the lattice constant were determined by the X-ray method except for that at the triple point, which was calculated from the density data of Clusius [7]. Both carbon resistors and the thermocouple were calibrated in place at the boiling point of helium, the triple point of hydrogen and the boiling point of hydrogen. In addition, readings from both resistors and the thermocouple were compared with the vapor pressure of hydrogen throughout the temperature range 10 to $20^{\circ} \mathrm{K}$. Temperature measurements are believed to be accurate to $\pm 0.1^{\circ} \mathrm{K}$ and lattice constants to $\pm 0.001 \mathrm{~A}$. The neon data demonstrate the importance of accurate control at temperatures below $20^{\circ} \mathrm{K}$. There are, of course, many other problems requiring temperature control that can be studied with this apparatus. For example, solid oxygen is known to exist in three forms, but, as far as we know, the structures of two of these and the effects of disorders that commonly occur, are still subjects for further study. It is hoped that this cryostat will contribute to the solution of these and other problems in the structure of low temperature solids.

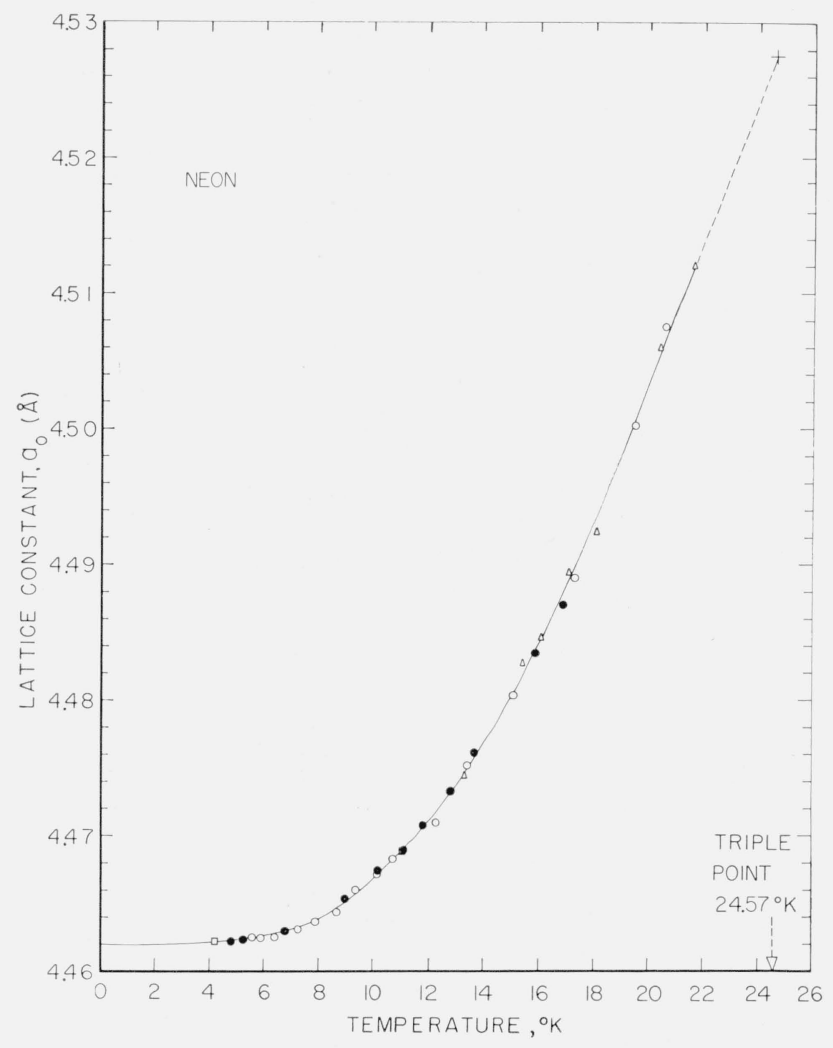

FIGURE 4. The lattice constant of neon as a function of temperature, showing the reproducibility between three experiments.

The value at $4.2^{\circ} \mathrm{K}$ is the average of four measurements. That at the triple point is calculated from the density reported by Clusius. 


\section{References}

[1] I. A. Black, L. H. Bolz, F. P. Brooks, F. A. Mauer, and H. S. Peiser, A liquid-helium cold cell for use with an X-ray diffractometer, J. Research NBS 61, 367 (1958) RP2907.

[2] D. G. Henshaw, Atomic distribution in liquid and solid neon and solid argon by neutron diffraction, Phys. Rev. 111, 1471 (1958)

[3] R. S. Hickman, R. W. Kenny, R. C. Mathewson, and R. A. Perkins, Liquid hydrogen targets of adhesivebonded Mylar plastic, Rev. Sci. Instr. 30, 983 (1959).

[4] R. D. Goodwin and J. R. Purcell, Direct-coupled power amplifier for cryostat heating control, Rev. Sci. Instr. 28, 581 (1957). (Twenty-six references are cited.)
5] J. R. Clement and E. H. Quinell, The low temperature characteristics of carbon-composition thermometers, Rev. Sci. Instr. 23, 213 (1952).

[6] L. H. Bolz and F A. Mauer, Lattice constants and thermal expansion of solid Ne, A, Kr, and Xe, to be submitted to J. Research NBS.

[7] K. Clusius, Atomwärmen und schmelzwärmen von Neon, Argon, und Krypton, Z. phys. Chem. B31, 459(1936).

(Paper 65C4-75) 